\title{
Efeito da radiação gama nas propriedades sensoriais, atividade de ógua e micobiota de arroz
}

\section{Effect of gamma radiation on sensorial properties, water activity and rice mycobiota}

\author{
Simone Aquino'; Thaise Cristine Fernandes Nunes'; Benedito Corrêa ${ }^{3}$ \\ ${ }^{1}$ Professora Doutora de Microbiologia e Micologia - Uninove, Curso de Farmácia. \\ São Paulo, SP - Brasil. \\ ${ }^{2}$ Mestre em Tecnologia Nuclear - IPEN/CNEN - SP, Centro de Tecnologia das Radiações/Aplicações. São Paulo, SP - Brasil
}

3Professor Titular - USP, Departamento de Microbiologia do ICB II/USP. São Paulo, SP - Brasil.

Endereço para correspondência

Simone Aquino - Universidade Nove de Julho - Uninove.

Av. Francisco Matarazzo, 613.

05001-100 - São Paulo - SP [Brasil]

siaq66@uninove.br

Local da pesquisa: Instituto de Ciências Biomédicas II - USP, Cidade de São Paulo, SP [Brasil].

\begin{abstract}
Resumo
Introdução: A contaminação fúngica ocorre frequentemente em arroz, após a colheita. Micotoxinas são metabólitos secundários produzidos por fungos toxigênicos considerados tóxicos para humanos e animais e causam injúria em órgãos-alvo, como câncer hepático. Objetivo: Neste estudo, objetivou-se determinar os efeitos da radiação gama na descontaminação fúngica, avaliar as alterações em propriedades sensoriais e nos valores de Atividade de água (Aa) dos grãos. Métodos: Amostras de arroz foram irradiadas com doses de 2 e 4 kGy e semeadas em ágar Batata e Dicloran Glicerol 18 \%. Resultados: Todos os grupos controle de arroz apresentaram diversos gêneros fúngicos, principalmente o grupo arroz integral, se comparado com o polido. A presença de fungos toxigênicos nas amostras representa um risco potencial na formação de micotoxinas. Conclusão: $\mathrm{O}$ tratamento nas doses de 2 e 4 kGy eliminou a contaminação fúngica, garantindo a qualidade do produto sem alterações significativas nas propriedades sensoriais.

Descritores: Arroz; Radiação gama; Fungos; Atividade de água.
\end{abstract}

\begin{abstract}
Introduction: Fungal contamination often occurs in rice after the harvest. Mycotoxins are secondary metabolites produced by toxigenic fungi that are considered toxic to humans and animals and cause serious target-organ injury, as hepatic cancer. Objective: The aim in this study was to determine the effect of gamma radiation on fungal decontamination, evaluate changes on the sensorial properties and on the water activity (Aw) values of grains. Methods: Samples of rice were irradiated at dose of 2 and $4 \mathrm{kGy}$ and in Potato and Dichloran glycerol agar. Results: All control groups of the samples were contaminated with different genera of fungi, mainly husked rice, comparing to polished rice. The presence of toxigenic fungi in samples represents a potential risk to mycotoxins forming. Conclusion: The treatment with dose of 2 and $4 \mathrm{kGy}$ eliminated the fungal contamination, ensuring the product quality without significantly changes in sensorial properties.
\end{abstract}

Key words: Rice; Gamma radiation; Fungi; Water activity. 


\section{Introdução}

O armazenamento de grãos sob condições não favoráveis são promotores do desenvolvimento de fungos e durante seu desenvolvimento esses produzem metabólitos tóxicos conhecidos como micotoxinas, que afetam a saúde humana e dos animais que os consomem. Estudos prévios já demonstraram que o arroz pode estar contaminado com micotoxinas ${ }^{1}$. Weidenboerner ${ }^{2}$ demonstrou a presença de aflatoxinas de $\mathrm{B}_{1}, \mathrm{~B}_{2} \mathrm{e}$ $\mathrm{G}_{1}$ em duas de cinquenta amostras de arroz polido no Brasil, numa concentração que variou de 26 a 96,3 $\mu \mathrm{g} / \mathrm{kg}$. Considerando as propriedades ecológicas do plantio, o crescimento dos fungos em arroz ocorre quando as concentrações de água nos grãos estão acima de 14,6 \% e, por essa razão, algumas espécies desses organismos podem crescer no início da colheita, quando o arroz ainda está úmido ${ }^{3}$. De acordo com Manabe e Tsuruta ${ }^{4}$, os fungos, em sua maioria, são mesófilos, ou seja, se desenvolvem em temperaturas medianas na faixa de 22 a $35^{\circ} \mathrm{C}$, mas são capazes de crescer também na de 5 a $45^{\circ} \mathrm{C}$.

As condições de umidade e temperatura no Brasil são características de países tropicais e subtropicais e apresenta condições ideais para o desenvolvimento de fungos em culturas. Muitos trabalhos validaram o emprego da radiação ionizante no controle de insetos, além do aumento da vida de prateleira de diversos produtos. Vários autores também já demonstraram que o emprego da radiação gama pode prevenir ou reduzir a contaminação fúngica.

Aziz e El-Aal ${ }^{5}$ reportaram que a completa eliminação de fungos toxigênicos em grãos de café e outros produtos foi alcançada com as doses de 5 a 10 kGy. Em estudos posteriores, Aziz et al. ${ }^{6}$ observaram que a dose requerida para uma completa inibição de fungos contaminantes em diversos tipos de alimentos variou de 4 a 6 kGy. A variação dos efeitos em relação à dose empregada pode mudar conforme o substrato irradiado. De acordo com Pitt e Miscamble ${ }^{7}$ o binômio entre Atividade de água (Aa) e temperatura ideal para que ocorra o crescimento fúngico e consequente produção de micotoxinas está em torno de $0,82\left(25{ }^{\circ} \mathrm{C}\right), 0,81\left(30{ }^{\circ} \mathrm{C}\right)$ e $0,80\left(37{ }^{\circ} \mathrm{C}\right)$. Entretanto, não há estudos que correlacionem a Aa com a dose de radiação gama empregada no tratamento para descontaminação de grãos de arroz. Aquino ${ }^{8}$ demonstrou a redução dos valores de Aa em grãos de guaraná irradiados nas doses de 5 e 10 kGy, quando comparados aos valores de amostras não irradiadas.

Segundo a legislação brasileira, o uso de métodos empregados na eliminação de contaminantes deve ser estudado a fim de prever qualquer alteração ou mudança na matéria-prima ${ }^{8}$. Alguns autores já relataram que o processo pelo tratamento por irradiação causou mudanças nas características de arroz do tipo integral, em termos de coloração, amilose, absorção de água, perdas de sólidos durante o cozimento, viscosidade, dureza (após o cozimento) e rancificação oxidativa ${ }^{9-11}$.

O objetivo neste estudo foi analisar a micobiota em cinco tipos de arroz, coletados no mercado varejista da cidade de São Paulo, avaliar a presença e eliminação da contaminação fúngica (empregando as doses de 2 e 4 kGy) e a redução da Aa do substrato e, consequentemente, possíveis alterações nas propriedades sensoriais das amostras irradiadas após o cozimento.

\section{Materiais e métodos}

\section{Amostras de arroz}

As amostras de cinco tipos de arroz (contendo $1 \mathrm{~kg}$ de cada tipo) foram adquiridas no comércio varejista da cidade de São Paulo e divididas em cinco grupos: agulhinha polido, integral com casca, integral sem casca, tailandês e parbolizado. Em seguida, foram fracionadas em sacos de polietileno em alíquotas de 100 gramas para os tratamentos e análises (Figura 1).

\section{Tratamento pelo processo de irradiação}

Amostras de arroz acondicionadas e vedadas em sacos de polietileno, contendo 100 
gramas cada, foram irradiadas com a dose de 2 e 4 kGy usando uma fonte de cobalto 60 (Gammacell 220) no Instituto de Pesquisas Energéticas e Nucleares (IPEN), localizado na cidade de São Paulo.

\section{Contagem da micobiota}

Após o processo de tratamento por irradiação, amostras irradiadas foram analisadas em comparação com amostras controle (não irradiadas). Os grãos (10 g) foram colocados em frascos contendo $90 \mathrm{ml}$ de água destilada estéril (primeira diluição) e agitados por 30 minutos em mesa agitadora horizontal. A amostra da primeira diluição foi submetida a diluições seriadas até $10^{-6} \mathrm{e}$, em seguida, uma suspensão de $0,1 \mathrm{ml}$ foi semeada por plaqueamento de superfície em duplicata. Dois meios de cultura foram utilizados: ágar Dicloran Glicerol 18\% (DG 18) e ágar Batata Dextrose (ABD). Após a incubação a $25{ }^{\circ} \mathrm{C}$ durante sete dias, a contagem (média da duplicata) foi expressa em unidades formadoras de colônias por grama (UFC/g).

\section{Determinação da atividade de ógua $(\mathrm{A} \propto)$}

A atividade de água (Aa) das amostras foi determinada por meio do uso de equipamento AQUALAB CX-2 (Decagon Devices), pertencente ao Laboratório de Micotoxinas do Instituto de Ciências Biomédicas II (USP).

\section{Teste de aceitação por escala hedônica}

As amostras de arroz (200 g) foram cozidas apenas em água e analisadas por 16 julgadores, que receberam (individualmente) fichas impressas nas quais anotaram suas avaliações. As amostras (identificadas apenas por números de três dígitos) foram avaliadas por meio de uma escala hedônica para determinar a preferência entre as amostras cozidas, considerando a nota um (1) como "não gostei", e a nove (9), como "gostei muito". Atributos sensoriais como aparência, odor e sabor foram analisados conjuntamente na mesma ficha. A análise estatística dos resultados foi realizada por meio do teste de múltipla comparação de Tukey e análise de variância ANOVA.

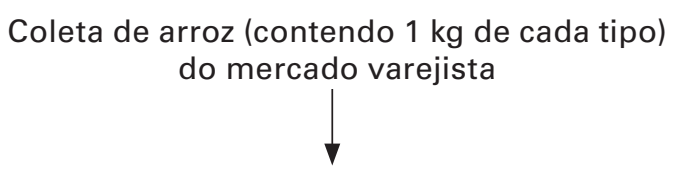

Fracionamento das amostras (100 gramas) e análise da micobiota

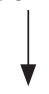

Análise da atividade de água das amostras de arroz

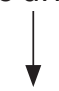

Tratamento por radiação ionizante (gama) nas doses de 2 e $4 \mathrm{kGy}$

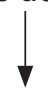

Análise da descontaminação fúngica após o tratamento por radiação

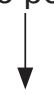

Análise da atividade de água das amostras de arroz após a irradiação

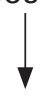

Análise sensorial das amostras irradiadas e grupo controle (não irradiado)

Figura l: Fluxograma experimental

\section{Resultodos}

\section{Isolomento fúngico}

Neste estudo, demonstrou-se que $80 \%$ das amostras cruas estavam contaminadas por bolores e leveduras, cuja contagem total variou de $0,5 \times 10^{2}$ a $5,0 \times 10^{5}$ nos grupos controle ou não irradiados (Tabelas 1 e 2), considerando os dois meios de cultura empregados (DG18 e ABD). Gêneros de fungos toxigênicos, como Aspergillus spp. e Penicillium spp., foram observados em 80 e $20 \%$, respectivamente, no grupo controle. As amostras de arroz integral com casca irradiadas 
com a dose de 2 kGy apresentaram o crescimento de fungo do gênero Alternaria. Nenhum crescimento fúngico foi observado em todos os tipos tratados com a dose de $4 \mathrm{kGy}$.

Tabela l: Contagens de gêneros fúngicos isolados dos grupos controle e tratados com as doses de 2 e $4 \mathrm{kGy}$, em meio DG 18

\begin{tabular}{|c|c|c|c|}
\hline Amostras* & Controle (0.0 kGy) & $2 \mathrm{kGy}$ & 4 kGy \\
\hline $\begin{array}{c}\text { Arroz } \\
\text { tailandês }\end{array}$ & $\begin{array}{c}\text { Aspergillus spp. } \\
\left(1,0 \times 10^{2}\right)\end{array}$ & $N D^{* * *}$ & $N D^{* * *}$ \\
\hline $\begin{array}{c}\text { Arroz } \\
\text { agulhinha } \\
\text { polido }\end{array}$ & ND & $N D^{* * *}$ & $N D^{* * *}$ \\
\hline $\begin{array}{c}\text { Arroz } \\
\text { integral } \\
\text { com casca }\end{array}$ & $\begin{array}{c}\text { Aspergillus spp. } \\
\left(3,5 \times 10^{2}\right) \\
\text { FNE } * * \\
\left(5,0 \times 10^{2}\right) \\
\text { Rhizopus spp. } \\
\left(2,0 \times 10^{2}\right) \\
\text { Cladosporium spp. } \\
\left(0,5 \times 10^{2}\right)\end{array}$ & $\begin{array}{c}N^{* * *} \\
\text { Alternaria spp. } \\
\left(1,5 \times 10^{2}\right)\end{array}$ & $\begin{array}{l}\mathrm{ND}^{* * * *} \\
\mathrm{ND}^{* * *}\end{array}$ \\
\hline $\begin{array}{c}\text { Arroz } \\
\text { integral } \\
\text { sem casca }\end{array}$ & $\begin{array}{c}\text { FNE }^{* *} \\
\left(4,0 \times 10^{2}\right) \\
\text { Penicillium spp. } \\
\left(1,5 \times 10^{2}\right) \\
\text { Aspergillus spp. } \\
\left(2,5 \times 10^{2}\right) \\
\text { Absidia spp. } \\
\left(0,5 \times 10^{2}\right)\end{array}$ & $N D^{* * *}$ & $N D^{* * *}$ \\
\hline $\begin{array}{c}\text { Arroz } \\
\text { parbolizado }\end{array}$ & $\begin{array}{c}\text { Cladosporium spp. } \\
\left(0,5 \times 10^{2}\right) \\
\text { Aspergillus spp. } \\
\left(1,0 \times 10^{2}\right) \\
\text { Rhodotorula spp. } \\
\left(0,5 \times 10^{2}\right)\end{array}$ & $N D * * *$ & $\mathrm{ND}^{* * *}$ \\
\hline
\end{tabular}

*Média de duplicata

$\mathrm{FNE}^{* *}$ - Fungo não esporulado

ND*** - Não detectado

O isolamento em meio ABD do grupo controle demonstrou que, com exceção de amostras de arroz tailandês, todas as amostras estavam contaminadas por fungos (Tabela 2). Foi observado também o crescimento de leveduras em arroz polido e integral, além da presença do fungo toxigênico Aspergillus spp. em $60 \%$ do grupo controle. As amostras tratadas com a dose de 2 kGy apresentaram crescimento de fungos filamentosos e leveduras, exceto a do arroz tailandês. Nenhum fungo foi observado nas amostras tratadas com a dose de 4 kGy. A dose de 2 kGy, aplicada neste estudo, não evitou o crescimento
Tabela 2: Contagem total em UFC/g de fungos contaminantes do grupo controle, $2 \mathrm{e}$ $4 \mathrm{kGy}$ em meio ABD

\begin{tabular}{|c|c|c|c|}
\hline Amostras* & Controle (0 kGy) & 2 kGy & 4 kGy \\
\hline $\begin{array}{c}\text { Arroz } \\
\text { tailandês }\end{array}$ & $N D^{* * *}$ & $N D^{* * *}$ & $N D^{* * *}$ \\
\hline $\begin{array}{c}\text { Arroz } \\
\text { agulhinha } \\
\text { polido }\end{array}$ & $\begin{array}{l}\text { FNE }^{* *} \\
\left(1,0 \times 10^{2}\right)\end{array}$ & $\begin{array}{c}\text { FNE** }^{* *} \\
\left(0,5 \times 10^{2}\right)\end{array}$ & $\mathrm{ND}^{* * *}$ \\
\hline $\begin{array}{c}\text { Arroz } \\
\text { integral } \\
\text { sem casca }\end{array}$ & $\begin{array}{c}\text { Curvularia spp. } \\
\left(0,5 \times 10^{2}\right) \\
\text { Scopulariopsis } \\
\text { spp. }\left(1,5 \times 10^{2}\right) \\
\text { Aspergillus spp. } \\
\left(0,5 \times 10^{2}\right)\end{array}$ & $\begin{array}{c}\text { Scopulariopsis } \\
\text { spp. } \\
\left(0,5 \times 10^{2}\right)\end{array}$ & $N D^{* * *}$ \\
\hline $\begin{array}{c}\text { Arroz } \\
\text { integral } \\
\text { com casca }\end{array}$ & $\begin{array}{c}\text { Aspergillus spp. } \\
\left(1,0 \times 10^{2}\right) \\
\text { Absidia spp. } \\
\left(1,0 \times 10^{2}\right) \\
\text { Rhizopus spp. } \\
\left(1,0 \times 10^{4}\right) \\
\text { Rhodotorula spp. } \\
\left(5,0 \times 10^{5}\right)\end{array}$ & $\begin{array}{c}\text { Rhodotorula spp. } \\
\left(6,8 \times 10^{5}\right) \\
\text { Absidia spp. } \\
\left(0,5 \times 10^{2}\right)\end{array}$ & $N D^{* * *}$ \\
\hline $\begin{array}{c}\text { Arroz } \\
\text { parbolizado }\end{array}$ & $\begin{array}{c}\text { FNE }^{* *} \\
\left(5,0 \times 10^{2}\right) \\
\text { Scopulariopsis } \\
\text { spp. }\left(1,0 \times 10^{3}\right) \\
\text { Aspergillus spp. } \\
\left(1,0 \times 10^{2}\right)\end{array}$ & $N D^{* * *}$ & $N D^{* * *}$ \\
\hline
\end{tabular}

de bolores (Alternaria spp., Scopulariopsis spp. e Absidia spp.) e leveduras (Rhodotorula spp.).

\section{Atividade de água (Aa)}

Os valores de Aa nas amostras controle variaram de 0,58 a 0,63, apresentando um decréscimo com o aumento das doses, após o tratamento (Figura 2). Já os valores das amostras não irradiadas demonstraram uma diferença significativa, quando comparadas com as amostras irradiadas nas doses de 2 kGy $(p<0,05)$ e 4 kGy $(p<0,01)$. As amostras irradiadas (2 e 4 kGy) não apresentaram diferença significativa entre si $(p>0,05)$.

\section{Anólise sensorial}

A cor amarelada dos grãos crus de arroz agulhinha polido, tailandês e parbolizado foi 


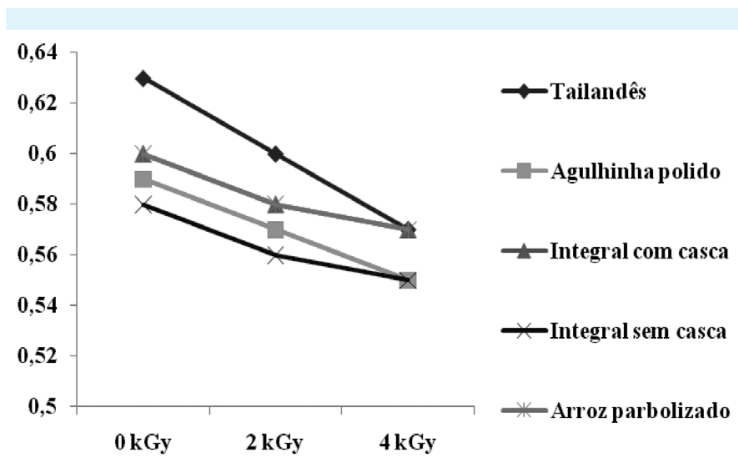

Figura 2: Valores de atividade de água (Aa) de amostras de arroz do grupo controle (0 kGy) e irradiadas (2 e 4 kGy)

observada nas amostras tratadas com a dose de $4 \mathrm{kGy}$, por $25 \%$, $18 \%$ e $6 \%$ dos julgadores, respectivamente, que não observaram em arroz integral com ou sem casca, quando comparadas ao grupo controle. Após o cozimento das amostras controle e irradiadas (mantendo as mesmas condições de volume de água e tempo de cozimento), 25 e $31 \%$ consideraram macias as amostras irradiadas com as doses de 2 e 4 kGy, nessa ordem. A classificação, segundo o teste de aceitação, demonstrou que $87 \%$ dos julgadores gostaram muito do arroz do grupo controle e $81 \%$ das amostras irradiadas com 2 kGy e 4 kGy, igualmente. Apenas 6\% acharam ambas as irradiadas, mais "borrachudas" na mastigação e observaram ainda que, o grão cozido estava mais "quebrado". Quanto ao sabor, 12\% atribuíram gosto e odor de "queimado" nas amostras cozidas irradiadas com 4 kGy. Entretanto, as pontuações para odor, aparência e gosto para amostras irradiadas (com 2 e 4 kGy) e não irradiadas não apresentaram diferenças significativas $(\mathrm{p}>0,05)$.

\section{Discussão}

\section{Isolamento fúngico}

Alguns dos gêneros isolados nesta amostragem já foram relatados em outros estudos. Hussaini et al. ${ }^{12}$ analisaram amostras de arroz coletadas na Nigéria e demonstraram que os gêneros fúngicos contaminantes presentes eram Aspergillus, Penicillium, Fusarium, Alternaria,
Mucor, Rhizopus, Trichoderma, Curoularia, Helminthosporium e Cladosporium. Uraguchi e Yamazaki ${ }^{13}$ também reportaram a presença de Aspergillus, Penicillium, Fusarium, Phoma, Curvularia, Helminthosporium, Cladosporium e Alternaria em arroz japonês. Penicillium, Aspergillus, Mucor e Rhizopus são fungos conhecidos como de armazenamento ${ }^{14,15}$. Penicillium, Aspergillus e Rhizopus germinam em determinadas condições de armazenamento em campos de regiões tropicais e subtropicais durante períodos de estiagem e devido à infestação de insetos. Entretanto, tais gêneros têm sido isolados de culturas de arroz recém colhidas ${ }^{14,16}$.

A presença de leveduras em amostras de arroz já foi descrita como fungo deteriorante de cereais e arroz ${ }^{17,15}$. O gênero Alternaria spp. requer umidade relativa e teores água elevados e, por isso, não é considerado competitivo em condições de armazenamento ${ }^{14}$, sendo comum em cereais no campo. Saleh et al. ${ }^{18}$ encontrou espécies de A. alternata, Cladosporium cladosporioides, Curvularia lunata e C. geniculata mais resistentes a radiação gama dentre dez espécies de fungos, representando o gênero Alternaria, Cladosporium, Curvularia, Fusarium e Penicillium. Aziz et al. ${ }^{6}$ mostraram que as contagens de micoflora viável diminuíram quase que exponencialmente com as doses de radiação; e a dose efetiva na eliminação de fungos foi 5 kGy para todas as plantas medicinais usadas em seu trabalho.

O fungo Alternaria alternata é conhecido por se desenvolver em ambientes com elevada radiação e já foi estudado como modelo para investigações genéticas a fim de explicar o fenômeno da radiorresistência. Um exemplo foi a sua presença em localidades poluídas com radioisótopos como o reator $\mathrm{n}^{\circ} .4 \mathrm{ChNPP}$ em Chernobyl ${ }^{19}$. Esse fungo é conhecido também por produzir e armazenar melanina, um pigmento negro, dentro de seu micélio. Existem indicações de que a radiorresistência de micro-organismos pode resultar da melanização de suas células ${ }^{20}$. Os mecanismos de reparo do Deoxyribonucleic Acid (DNA) são predominantemente responsáveis 
pela resistência à radiação e pode ter um papel importante na sobrevivência de fungos ${ }^{21}$.

\section{Atividade de ógua (Å)}

Os valores encontrados de Aa estão em uma faixa considerada segura para o crescimento e produção de micotoxinas no substrato. Sirisoontaralak e Noomhorm ${ }^{22}$ analisaram a qualidade do arroz no cozimento e observaram que um menor teor de água foi absorvido nas amostras controle, sendo um significativo aumento observado em tratamentos com doses acima de 1,5 kGy. Os autores concluíram que as proteínas e o amido se fragmentariam com a irradiação, causando uma elevação no número de pontos de ligações de água. Juliano ${ }^{23}$ elaborou uma teoria de que a irradiação poderia quebrar a matriz protéica ao redor dos grânulos de amido que serviria como uma barreira física à absorção de água. Chaudhry e Glew ${ }^{24}$ relataram que a irradiação poderia modificar os grânulos e permitir que a água penetrasse com relativa facilidade e causar danos físicos e, com isso, de acordo com Meredith ${ }^{25}$, a menor partícula de amido teria sua área de superfície aumentada para hidratação. Diehl ${ }^{26}$ relatou que a presença de água tem um importante papel no processo de irradiação, uma vez que a radiólise da água do substrato leva a formação de radicais livres altamente reativos (produtos dessa radiólise), quando a energia da radiação quebra as moléculas de água e, consequentemente, uma maior energia da radiação gama poderia resultar num aumento da formação de radicais livres (altamente reativos). Isso também poderia explicar a diminuição da água livre em grãos irradiados e esse efeito poderia influenciar na absorção de água pelo grão de arroz (mais seco quando irradiado), corroborando resultados observados neste estudo.

\section{Anó́lise sensorial}

Em relação à dose absorvida, a $\mathrm{RDC} \mathrm{n}^{\circ}$ 21, publicada em 26 de janeiro de 2001 pela
ANVISA $^{27}$, determina que não haja limite mínimo e máximo para o tratamento pelo processo de irradiação de qualquer alimento, desde que a dose mínima absorvida seja suficiente para alcançar a finalidade pretendida, e a máxima absorvida seja inferior àquela que comprometeria as propriedades funcionais e ou os atributos sensoriais do alimento. Portanto, em qualquer estudo em que se submeta um produto ao processo por irradiação, esse deve ser analisado considerando as alterações sensoriais (de acordo com as doses mínimas e máximas empregadas). O odor desagradável e sabor alterado podem ocorrer em razão da presença de produtos oriundos da degradação lipídica e devido à concentração de alquenos formados por autoxidação $^{28}$. Sirisoontaralak e Noomhorm ${ }^{29}$ compararam arroz não irradiado com irradiado e estocado por um ano e observaram menores pontuações para odor e sabor de amostras irradiadas em decorrência da formação de produtos da oxidação lipídica como peróxidos e compostos de carbonila (que surgem imediatamente após a irradiação). De acordo com os mesmos autores, efeitos adicionais em termos de hidrólise e oxidação lipídica natural pioram com o tempo de prateleira do arroz irradiado, inviabilizando a comercialização do produto no mercado varejista e atacadista.

\section{Conclusões}

Neste estudo, demonstrou-se que arroz integral (com ou sem casca) e arroz do tipo parbolizado apresentaram uma variedade de gêneros fúngicos, além de elevada contaminação, se comparados com o agulhinha polido e com o tailandês. A presença de fungos toxigênicos em arroz representa um risco potencial na formação de micotoxinas nesses grãos, em condições inadequadas de armazenamento. $\mathrm{O}$ tratamento pelo processo de irradiação elimina o crescimento fúngico, além do efeito adicional da redução da Aa, como demonstrado nas doses de 2 e 4 kGy. Entretanto, apenas o tratamento pelo 
processo de irradiação não garante a qualidade do produto, se não observadas as boas práticas de armazenamento. $\mathrm{O}$ tratamento por radiação ionizante na dose de 4 kGy, não causou mudanças significativas nas propriedades sensoriais imediatamente ao processo; entretanto, estudos complementares sobre as propriedades sensoriais, em períodos variáveis de armazenamento, são necessários para avaliar as reais condições da vida de prateleira no mercado varejista.

\section{Referências}

1. Tanaka K, Sago Y, Zheng Y, Nakagawa H, Kushiro M. Mycotoxins in rice. Int J Food Microbiol. 2007;119:59-66.

2. Weidenboerner M. Encyclopedia of food mycotoxins. Berlin: Springer-Verlag; 2000.

3. Uraguchi K. Mycotoxic Origin of Cardiac Beriberi. J Stored Prod Res. 1969;5:227-36.

4. Manabe M, Tsuruta O. Mycoflora and mycotoxins in stored rice grain. In: Chelkowski J editor. Cereal grain: mycotoxins, fungi and quality in drying and storage. Elsevier; 1991.

5. Aziz NH, Abd El-Aal SS. Occurrence of aflatoxin and aflatoxigenic molds in coffee beans and decontamination by gamma-irradiation. J Egypt Vet Med Ass. 1990;49:951-62.

6. Aziz NH, El-Fouly MZ, Abu-Shady MR, Moussa LAA. Effect of gamma radiation on the survival of fungal and actinomycetal florae contaminating medicinal plants. Appl Radiat Isot. 1997;48(1):71-6.

7. Pitt JI, Miscamble BF. Water relations of Aspergillus flavus and closely related species. J Food Protect. 1995;58:86-90.

8. Aquino S, Gonçalez E, Reis TA, Sabundjian IT, Trindade RA, Rossi MH, et al. Effect of gamma irradiation on mycoflora of guarana (Paullinia cupana). Radiat Phys Chem. 2007;76:1470-3.

9. Navanugraha U, Grant DR. Effect of gamma irradiation on the cooking characteristics of wild rice. Food Res Int. 1992;25:199-202.

10. Roy MK, Ghosh SK, Chatterjee SR. Gamma irradiation of rice grains. J Food Sci Technol. 1991;28:337-40.
11. Hayashi T, Okadome H, Toyoshima H, Todoriki S, Ohtsubo K. Rheological properties and lipid oxidation of rice decontaminated with low-energy electrons. J Food Protect. 1998;61:73-7.

12. Hussaini AM, Timothy AG, Olufunmilayo HA, Ezekiel AS, Godwin HO. Fungi and some mycotoxins contaminating rice (Oryza Sativa) in Niger State, Nigeria. African J Biotechnol. 2007;6(2):99-108.

13. Uraguchi K, Yamazaki M. Toxicology: biochemistry and pathology of mycotoxins. Japan; Halsted press; 1978.

14. Ominski KH, Marquardi RR, Sinha RN, Abramson D. Ecological aspects of growth and mycotoxin production by storage fungi. In: Miler JD and Trenholm HL editors. Mycotoxins in grains: Compounds other than aflatoxins. St. Paul Minnesota: Eagan Press; 1994. p. 287-314.

15. Taligoola H, Ismail MA, Chebon SK. Mycobiota associated with rice grains marketed in Uganda. J Biol Sci. 2004;4(1):271-8.

16. Garcia RP. Survey of mycoflora association with Azolla spp. The Philipine Agri. 1986;69:529-34.

17. Miroslava K. Feeding soybean colonization by microscopic fungi. Trakya Univ J Sci. 2003;4(2):165-8.

18. Saleh YG, Mayo MS, Ahearn DG. Notes: resistance of some common fungi to amma irradiation. Appl Environ Microbiol. 1998;54(8):21340-5.

19. Mironenko NV, Alekhina IA, Zhdanova NN, Bulat $\mathrm{SA}$. Intraspecific variation in gamma-radiation resistance and genomic structure in the filamentous fungus Alternaria alternata: a case study of strains inhabiting Chernobyl reactor $n^{\circ} .4$ Ecotox Environ Safety. 2000;45:177-87.

20. Pointing S, Jones EBG, Jones M. Radiosensitivity of fungi isolated from waterlogged archaeological wood. Mycoscience. 1996;37:455-8.

21. Boreham DR, Mitchel REJ. Heat and radiation stress response regulation in yeast by HSP104. Radiat Res. 1994;137:190-5.

22. Sirisoontaralak $\mathrm{P}$, Noomhorm A. Changes to physicochemical properties and aroma of irradiated rice. J Stored Prod Res. 2006;42:264-76.

23. Juliano BO. Criteria and tests for rice grain qualities. In: Juliano BO editor. Rice: Chemistry and Technology. St. Paul, Minnesota: The American Association of Cereal Chemists; 1985. p. 443-524. 
24. Chaudhry MA, Glew G. The effect of ionizing radiation on some physical and chemical properties of Pakistani rice. J Food Technol. 1973;8:295-303.

25. Meredith P. Dependence of water absorption of wheat on protein content and degree of starch granule damage. N Z J Sci. 1966;9:324.

26 Diehl JF. Safety of Irradiated Foods. New York: Marcel Dekker Inc; 1995. p. 91-115.

27. Brasil. Resolução RDC n 21 de 26 janeiro de 2001. Dispõe sobre regulamento Técnico para Irradiação de Alimentos. Agência Nacional de Vigilância Sanitária (ANVISA). Diário Oficial da União (D.O.U.). 200129 jan.
28. Yasumatsu K, Moritaka S, Kakinuma T. Effect of the change during storage in lipid composition of rice on its amylogram. Agr Biol Chem. 1964;28:265-72.

29. Sirisoontaralak P, Noomhorm A. Changes to physicochemical properties and aroma of irradiated rice. J Stored Prod Res. 2006;42:264-76. 